\title{
PRELIMINARY RESULTS FROM THE CHICAGO AIR SHOWER ARRAY AND THE MICHIGAN MUON ARRAY*
}

\author{
H.A. Krimm, J.W. Cronin, B.E. Fick, K.G. Gibbs, N.C. Mascarenhas, T.A. McKay, \\ D. Müller, B.J. Newport, R.A. Ong, L.J Rosenberg, M.E. Wiedenbeck
}

The Enrico Fermi Institute, The University of Chicago, Chicago, IL 60637 U.S.A.

K.D. Green, J. Matthews, D. Nitz, D. Sinclair, J.C. van der Velde

Department of Physics, The University of Michigan, Ann Arbor, MI 48109 U.S.A.

\begin{abstract}
The Chicago Air Shower Array (CASA) is a large area surface array designed to detect extensive air showers (EAS) produced by primaries with energy $\sim 100 \mathrm{TeV}$. It operates in coincidence with the underground Michigan Muon Array (MIA). Preliminary results are presented from a search for steady emission and daily emission from three astrophysical sources: Cygnus X-3, Hercules X-1, and the Crab nebula and pulsar. There is no evidence for a significant signal from any of these sources in the 1989 data.
\end{abstract}

\section{INTRODUCTION}

The Chicago Air Shower Array and the Michigan Muon Array are located at Dugway, Utah, U.S.A. $\left(40.2^{\circ} \mathrm{N}, 112.8^{\circ} \mathrm{W}\right)$ at an atmospheric depth of $870 \mathrm{~g} \cdot \mathrm{cm}^{-2}$. Detailed descriptions of $\mathrm{CASA}^{1,2}$ and $\mathrm{MIA}^{3}$ can be found elsewhere. From 1 February 1989 to 30 November 1989, CASA operated with 49 detector stations, each containing $1.5 \mathrm{~m}^{2}$ of plastic scintillator, arranged on a square grid of total area $8100 \mathrm{~m}^{2}$. MIA consisted of 512 buried counters of area $2.5 \mathrm{~m}^{2}$ distributed in eight widely separated patches. The overall event rate was $1.2 \mathrm{~Hz}$ over 232.6 days of live time, rendering 24.6 million total events. Due to its low threshold, the array triggers on some showers that are too small to permit proper reconstruction. The analysis was based on 22.0 million events with shower size $N_{e} \geq 1000$ particles and reconstructed zenith angle $\theta \leq 60^{\circ}$. CASA/MIA provides an estimate of the number of muons in the shower, $\mathrm{N}_{\mu}$, and the total shower size, allowing a cut on muon-poor events. Accurate shower size reconstruction, however, requires the core to fall well within the array, a stringent cut which reduces the data set to 7.8 million events of which $\mathbf{5 . 5}$ million have muon information. The muon size cut is described in detail below.

\section{EVENT RECONSTRUCTION}

The first stage of high level reconstruction of CASA events is the determination of the shower size. A lateral density profile is calculated based on the number of particles detected in each CASA station, and this profile is fit to the empirical function developed by Nishimura, Kamata and Greisen, ${ }^{4}$ using an age of 1.28 and Moliere radius of 64 meters. The average shower size is found to be $\sim 24,800$ particles and the data is represented by the relation:

$$
\mathrm{N}_{\mathrm{e}}=470 \cdot\left(\mathrm{n}_{\mathrm{e}}\right)^{0.91} \quad\left(\mathrm{~N}_{\mathrm{e}}>10^{4}\right),
$$

where $n_{e}$ is the actual number of particles sampled by the array.

Muon data is collected separately by MIA and merged with CASA on an event-by-event basis. An average shower produces 13.4 muon hits, including 0.6 accidentals. If an event has at

'Proc. Int. Conf. on High Energy Gamma-Ray Astronomy, Oct. 2-5, 1990, Ann Arbor, MI. 
least two muon hits, a Greisen function ${ }^{5}$ with age of 1.28 and scale length 300 meters is used to fit the muon lateral density distribution. The data can be parameterized by the function:

$$
\mathrm{N}_{\mu}=230 \cdot\left(\mathrm{n}_{\mu}\right)^{1.03} \quad\left(\mathrm{~N}_{\mu}>10^{3}\right)
$$

where $\mathrm{n}_{\mu}$ is the total number of muon hits. The average reconstructed muon size is 4600 particles.

The muon size and total shower size are found to be related by Equation 3 which was fit to data with $\mathrm{N}_{\mathrm{e}}>10^{4}$ :

$$
\log _{10}\left\langle\mathrm{~N}_{\mu}\right\rangle=-0.62+0.54 \cdot \sec \theta+0.78 \cdot \log _{10}\left(\mathrm{~N}_{\mathrm{e}}\right) .
$$

The zenith angle dependence arises from the dissimilar development of the electron and muon components of an extensive air shower as a function of depth.

For each event, Equation 3 is used to calculate the expected muon size $\left\langle\mathrm{N}_{\mu}\right\rangle$ which is compared with that observed, $N_{\mu}$. Simulations of EAS ${ }^{6}$ affirm that, at the energies of interest for CASA, the muon content of a gamma ray induced shower is diminished by a factor of more than thirty relative to a hadron induced shower of the same energy. Thus if a shower produces less than one tenth the expected number of muons or if $\mathrm{n}_{\mu} \leq 1$, the shower is considered to be muon poor and hence a gamma ray candidate. Figure 1 displays the relationship between $\left\langle\mathrm{N}_{\mu}\right\rangle$ and $\mathrm{N}_{\mu}$. The hadron rejection factor is approximately eleven for all showers, increasing with shower size to more than fifty for showers with $N_{e}>25,000$.

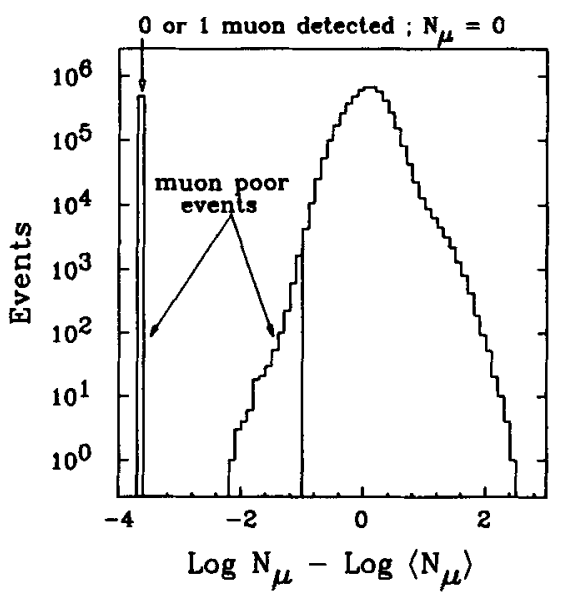

Figure 1. Distribution of relative muon sizes for events with cores falling within the array. $\left\langle\mathrm{N}_{\mu}\right\rangle$ is defined by Equation 3.

\section{DETERMINATION OF THE ENERGY SCALE}

To determine a flux level from an observation, it is necessary to understand the energy scale of the experiment. For this array, the spectrum is derived by first noting that the atmospheric depth through which an EAS must progress increases with $\theta$. Since CASA is well below shower maximum for all but the most energetic EAS, the observed size of a shower derived from a primary of a given energy decreases with increasing overburden. Conversely, the energy of the primary required to produce a shower of a particular size rises with $\theta$. Since CASA is sensitive to showers from a wide range of zenith angles $\left(0^{\circ}<\theta<60^{\circ}\right)$, one can generate Figure 2, a series of curves, each for a different shower size, relating observed integral flux to atmospheric overburden. A line of constant flux in this figure corresponds to a specific energy, the correspondence depending on the choice of spectral index and overall flux normalization. Using Protheroe's parameterization $^{7}$ of the cosmic ray energy spectrum compiled by Hillas ${ }^{8}$ and the information contained in Figure 2, one can derive the energy scale of EAS observed by CASA. Figure 3 shows this spectrum with a mean observed energy of $198 \mathrm{TeV}$ and median energy $136 \mathrm{TeV}$. 


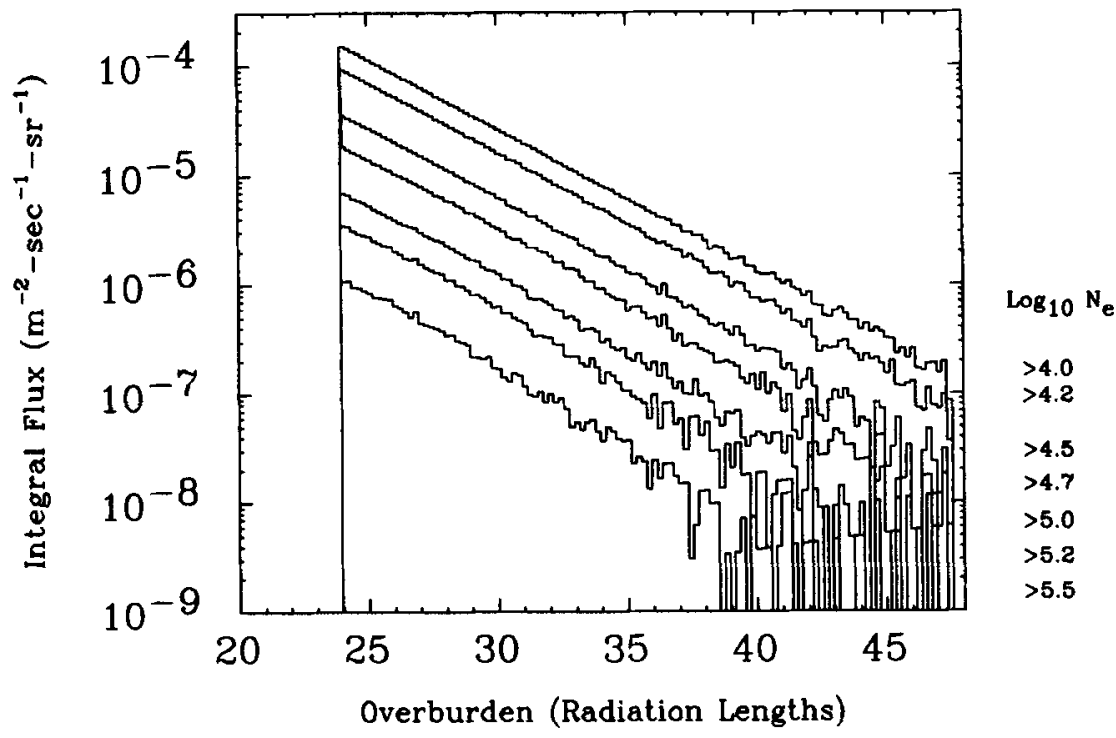

Figure 2. The integral flux of cosmic rays observed by CASA as a function of atmospheric overburden. Each curve corresponds to a different shower size. Refer to the text for details.

\section{SEARCH FOR STEADY EMISSION FROM ASTROPHYSICAL SOURCES}

Using the data from 1989, a search was undertaken for steady (d.c.) emission from three astrophysical objects (Table I) which have been reported as possible sources of ultra-high energy (UHE) garnma rays. The search for excess events was carried out on the full data set and on two subsets of the data, both of which yield lower flux limits due to improved background rejection. Characteristics of these three data sets are listed in Table II. The angular resolution $\sigma_{\theta}$ is defined as the half angle of a cone which would contain $63 \%$ of the reconstructed showers from the direction of a point source; the cuts used to obtain the muon poor data are discussed in Section II. Signal candidates were those falling into a rectangular bin centered on the source with size $\Delta_{\text {decl }} \times\left[\Delta_{\text {decl }} / \cos (\operatorname{decl})\right]$, where $\Delta_{\text {decl }}$ is given in Table II. The background was determined from the data, based on a daily calculation of the acceptance of the array as a function of the angular position of the source.

Table III lists the number of observed and expected background events from each source direction summed over the year's run, as well as flux limits calculated assuming a Gaussian distribution of background fluctuations. The effective area and detection efficiency were derived from the observed background rate and the parameterized cosmic ray flux. The limits presented are conservative because the gamma ray spectrum is assumed to have the same form as the background and be-

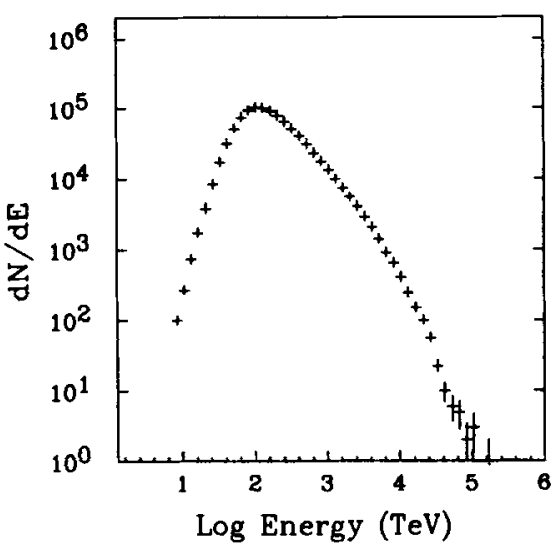

Figure 3. The CASA energy scale assuming that all showers are due to hadrons. 
Table I: Three potential sources of UHE gamma rays

Values given are for a square search bin of $2.6^{\circ} \times 2.6^{\circ} / \cos (\operatorname{decl})$

\begin{tabular}{||l|c|c|c||}
\hline Source: & Cygnus X-3 & Hercules X-1 & Crab \\
\hline Minimum zenith angle at $40.2^{\circ} \mathrm{N}:$ & $0.59^{\circ}$ & $3.56^{\circ}$ & $16.91^{\circ}$ \\
Approximate daily transit time: & $11 \mathrm{~h} 3 \mathrm{~m}$ & $10 \mathrm{~h} 31 \mathrm{~m}$ & $9 \mathrm{~h} 14 \mathrm{~m}$ \\
$\begin{array}{l}\text { Number of full transits: } \\
\begin{array}{l}\text { Average daily number } \\
\text { of background events (all data): }\end{array}\end{array}$ & 223 & 227 & 221 \\
\hline
\end{tabular}

Table II: Overview of the three data sets used in the searches

\begin{tabular}{||l|c|c|c||}
\hline & All data & $\begin{array}{c}\text { Size cut data } \\
\left(\mathrm{N}_{\mathrm{e}}>25,000\right)\end{array}$ & $\begin{array}{c}\text { Muon poor } \\
\text { data }\end{array}$ \\
\hline Angular resolution $\sigma_{\theta}:$ & $1.2^{\circ}$ & $0.8^{\circ}$ & $1.2^{\circ}$ \\
Bin size in declination $\Delta_{\text {decl }}:$ & $2.6^{\circ}$ & $1.6^{\circ}$ & $2.6^{\circ}$ \\
Total events: & $22,010,000$ & $5,362,000$ & 494,000 \\
\hline
\end{tabular}

Refer to the text for definitions

Table III: Integral flux limits (E > $100 \mathrm{TeV}$ ) from D.C. searches

\begin{tabular}{||c|c|c|c|c||}
\hline Source & Data set & $\begin{array}{c}\text { Observed } \\
\text { events }\end{array}$ & $\begin{array}{c}\text { Expected } \\
\text { background }\end{array}$ & $\begin{array}{c}\text { Flux }(95 \% \text { C.L.) } \\
\left(\mathrm{cm}^{-2} \cdot \mathrm{sec}^{-1}\right)\end{array}$ \\
\hline \multirow{3}{*}{ Cygnus X-3 } & all data: & 12,145 & 12,197 & $<4.3 \times 10^{-13}$ \\
& $\mathrm{~N}_{\mathrm{e}}>25,000:$ & 1112 & 1127 & $<5.3 \times 10^{-13}$ \\
& muon poor: & 270 & 275 & $<2.6 \times 10^{-13}$ \\
\hline \multirow{3}{*}{ Hercules X-1 } & all data: & 11,867 & 11,776 & $<6.6 \times 10^{-13}$ \\
& $\mathrm{~N}_{\mathrm{e}}>25,000:$ & 1147 & 1098 & $<10.5 \times 10^{-13}$ \\
& muon poor: & 273 & 270 & $<3.0 \times 10^{-13}$ \\
\hline \multirow{3}{*}{ Crab } & all data: & 6669 & 6696 & $<5.9 \times 10^{-13}$ \\
& $\mathrm{~N}_{\mathrm{e}}>25,000:$ & 602 & 612 & $<7.3 \times 10^{-13}$ \\
& muon poor: & 147 & 152 & $<3.5 \times 10^{-13}$ \\
\hline
\end{tabular}

cause the calculations do not take into account the prediction that gamma rays will produce larger showers than hadrons of the same energy.

The data were further analyzed for evidence of daily excesses from the three sources. The result of such a search in the direction of Cygnus X-3 is shown in Figure 4. The plot also indicates the days on which radio outbursts were reported from Cygnus X-3.

Figure 5 confirms that the daily significances do not deviate from what would be expected from a Gaussian distribution with zero mean and unit standard deviation. Similar results were obtained for Hercules X-1 and the Crab. 


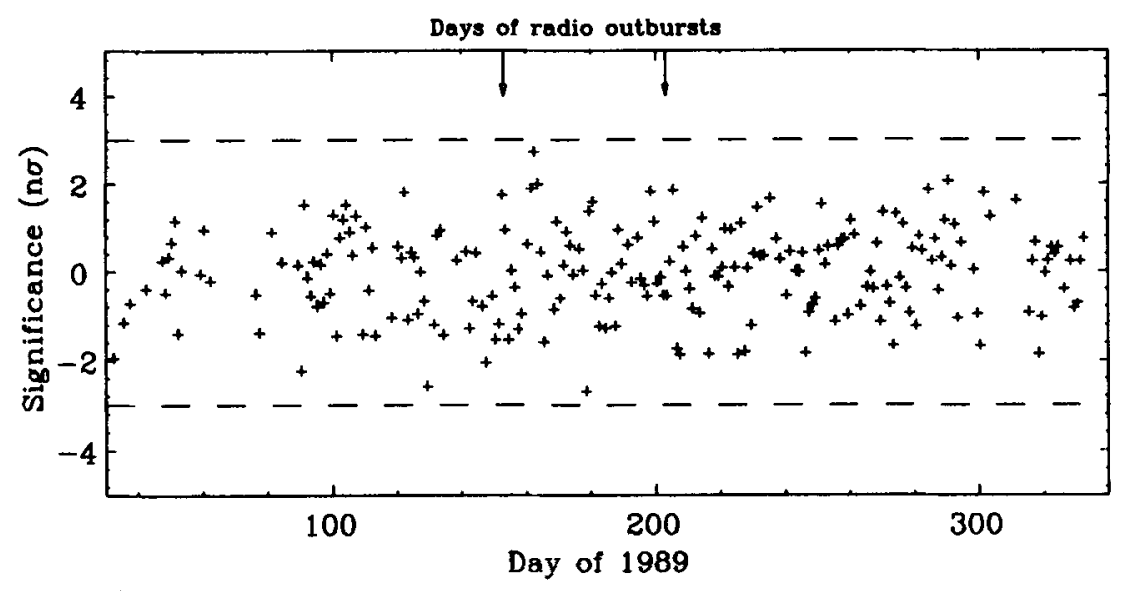

Figure 4. The statistical significance of daily signals from the direction of Cygnus X-3, calculated using the statistics discussed by $\mathrm{Li}$ and $\mathrm{Ma}^{9}$. A $3 \sigma$ excess would correspond to a flux of $\sim 1.1 \times 10^{-11} \mathrm{~cm}^{-2} \cdot \mathrm{sec}^{-1}$.

\section{CONCLUSIONS AND OUTLOOK}

Preliminary analysis of the 1989 data from the Chicago Air Shower Array shows no evidence for steady gamma ray emission from Cygnus X-3, Hercules X-1, or the Crab nebula and pulsar. The flux limits obtained for these sources are listed in Table III. Further analysis of this data set will include a search for periodic source emission. A significant improvement in sensitivity will be realized when CASA begins operation with its full complement of 1089 detector stations.

We gratefully acknowledge the help and support of the members of the University of Utah Fly's Eye Collaboration and of Colonel Van Prooyen and the staff of the Dugway Proving Ground. This work is supported in part by the National Science Foundation and the U.S Department of Energy.

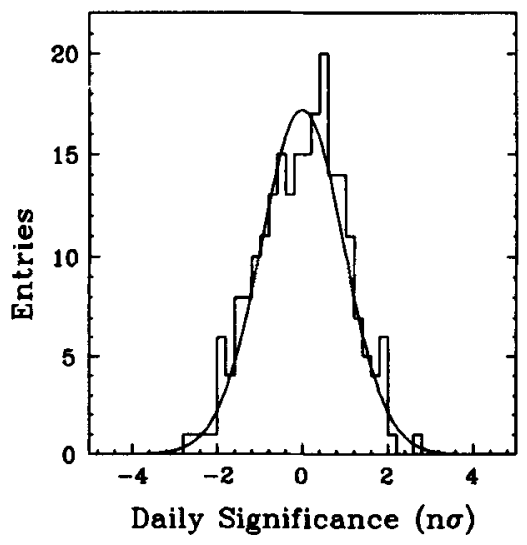

Figure 5. The accumulation of daily significances from Figure 4. The solid line is a fit to a Gaussian distribution with mean -0.0119 and standard deviation 0.987 .

\section{REFERENCES}

1. R.A. Ong, Nucl. Phys. B (Proc. Suppl.) 14A, 273, (1990).

2. L.J Rosenberg et. al., This Conference.

3. D. Sinclair, Nucl. Instrum. Methods A278, 583, (1989).

4. K. Greisen, Annu. Rev. Nucl. Sci. 10, 63, (1960), eq. 3.3.

5. K. Greisen, op. cit., eq. 4.1.

6. T. Stanev, T.K. Gaisser, and F. Halzen, Phys. Rev. D 32, 1244, (1985).

7. R.J. Protheroe, Proc. 20th Int. Cosmic Ray Conf., Moscow, 8, 21, (1987).

8. A.M. Hillas, Proc. Cosmic Ray Workshop, Univ, of Utah, ed. T.K. Gaisser (Bartol Res. Fdn., Newark), 16, (1983).

9. T. Li and Y. Ma, Astrophys. J., 272, 317, (1983). 